\title{
Brucelosis Bovina en la Provincia Manabí, Ecuador. Estudio de los Factores de Riesgo
}

\author{
Bovine Brucellosis in the Manabí Province, Ecuador. Study of Risk Factors \\ Marina Dalila Zambrano Aguayo ${ }^{1,4}$, Miguel Pérez Ruano², \\ Ximena Rodríguez Villafuerte ${ }^{3}$
}

\section{Resumen}

\begin{abstract}
Se realizó un estudio epidemiológico transversal para determinar los factores de riesgo de los animales y de los hatos asociados a brucelosis bovina. Se seleccionaron 2369 bovinos de la provincia Manabí, Ecuador; asimismo, se realizó un estudio epidemiológico de tipo ecológico, donde se seleccionaron 20 hatos positivos (casos) y 20 hatos negativos (controles) a brucelosis. En ambos estudios se aplicó una encuesta epidemiológica para determinar los principales factores de riesgo asociados a la presentación de la enfermedad. El estudio individual determinó un mayor riesgo de padecer la enfermedad para los animales mayores de 5 años $(p<0.0001)$ y para hembras $(p<0.05)$. En los hatos, las ganaderías dedicadas a la producción de leche $(\mathrm{p}<0.05)$, las que no investigan en ganado antes de ingresar a sus predios $(p<0.05)$ y las que no vacunan $(p<0.001)$ presentan un mayor riesgo de afectarse por la enfermedad. Se comprobó, además, una mayor probabilidad de presentación de abortos en ganaderías afectadas por brucelosis que en las no afectadas $(\mathrm{p}<0.02)$. Se concluye que hay un mayor riesgo a presentar la enfermedad en animales mayores de 5 años y en fincas dedicadas a la producción de leche, en las que no investigan el estado sanitario de los bovinos que ingresan a la finca y en las que no vacunan contra la enfermedad.
\end{abstract}

Palabras clave: Brucella sp, abortos, estudio epidemiológico, factores de riesgo, Ecuador

\footnotetext{
${ }^{1}$ Departamento de Medicina Veterinaria, Facultad de Ciencias Veterinarias, Universidad Técnica de Manabi (UTM), Portoviejo, Ecuador

${ }^{2}$ Departamento de Medicina Preventiva, Facultad de Medicina Veterinaria, Universidad Agraria de la Habana (UNAH), Mayabeque, Cuba

${ }^{3}$ Departamento del Área del Conocimiento de la Tierra, Carrera de Ingeniería Agropecuaria, Universidad Estatal Amazónica (UEA), Puyo, Ecuador

${ }^{4}$ Email: mazambrano@utm.edu.ec
}

Recibido: 3 de diciembre de 2015

Aceptado para publicación: 20 de marzo de 2016 
A cross-sectional epidemiological study was carried out to determine animal and herd risk factors associated to bovine brucellosis. It was randomly selected 2369 cattle in the Manabí province, Ecuador. In addition, an epidemiological study of ecological type was carried out using 20 positive herds (cases) and 20 negative herds (controls) to the disease. In both studies, an epidemiological survey was conducted to determine the main risk factors associated to the disease. The individual study showed a higher risk of having the disease in animals older than 5 years $(p<0.0001)$ and in females $(p<0.05)$. Herds involved in milk production $(\mathrm{p}<0.05)$, those that do not check the aniamls before entering the premises $(p<0.05)$ and those without vaccination $(p<0.001)$ had a higher risk of being affected by the disease. It was also demonstrated that there was a greater probability of having abortions in infected herds than in those unaffected $(p<0.02)$. It is concluded that there was an increased risk of presenting the disease in animals over 5 years old, in dairy herds and in those that do not check the health status of animals prior to the introduction to the farm and in those without vaccination.

Key words: Brucella sp, abortions, epidemiological study, risk factors, Ecuador

\section{INTRODUCCIÓN}

La brucelosis bovina se produce fundamentalmente por la presencia de la bacteria Brucella abortus (Díaz, 2013). Esta bacteria causa infecciones crónicas tanto en el hombre como en el ganado vacuno (RonRomán et al., 2014).

La transmisión entre animales se produce por ingestión de pastos, alimentos y agua contaminados con excreciones, a través de membranas fetales de vacas infectadas y secreciones vaginales que pueden ingresar por vía ocular e incluso a través de la piel indemne de animales estabulados, por contacto con fetos abortados y machos infectados, y por inseminación artificial realizada sin considerar las adecuadas medidas higiénicas. En el semen de machos infectados se encuentra la bacteria, de allí que el uso del semen mediante la monta directa o a través de la inseminación artificial es una importante vía de infección a hembras libres de brucelosis (AGROCALIDAD, 2009).

La transferencia de embriones no tratados en forma adecuada puede constituir una fuente adicional de infección. Asimismo, fincas libres pueden ingresar la infección a través del contacto de animales sanos con animales infectados en ferias o eventos ganaderos donde participen animales de diversos lugares, por la compra de animales portadores de la infección, así como por el ingreso de animales infectados de otras especies (Ministerio de Agricultura y Desarrollo Rural e Instituto Colombiano Agropecuario, 2010).

Estudios realizados en Ecuador sobre la situación de la enfermedad indican una alta prevalencia de la enfermedad; asimismo, reportan el tamaño de los rebaños como uno de los principales factores de riesgo para la presentación de la enfermedad (Poulsen et al., 2014). En otro estudio, llevado a cabo en la provincia ecuatoriana de Carchi (Ayala y Tobar, 2011), indican que la prevalencia de la brucelosis bovina está directamente relacionada con la densidad de la población del ganado. Asimismo, se han reconocido como factores de riesgo en el país la introducción como reemplazo de animales procedentes de ferias, falta de inmunidad de los animales, el tipo de reproducción y la falta de pediluvios en la entrada de las fincas (Neppas, 2013). 
Otros autores (Borba et al., 2013) señalan como factores de riesgo la edad de los animales, el clima, el pastoreo en áreas contaminadas, la presencia de áreas inundadas, la inseminación con semen infectado y el consumo de aguas contaminadas.

La provincia de Manabí, Ecuador, presenta el mayor censo ganadero del país (Instituto Nacional de Estadísticas y Censo, 2012), y si bien se han realizados estudios parciales vinculados fundamentalmente a tesis de grado, no existen estudios científicos publicados que permitan conocer los principales factores de riesgo para la presentación de brucelosis en el ganado bovino, de allí que el presente estudio tuvo como objetivo determinar los principales factores de riesgo asociados a la presentación de la brucelosis bovina en esta provincia.

\section{Materiales y Métodos}

\section{Área Experimental}

El área experimental se sitúa en la provincia Manabí, con un área de 18893.7 km², representando el $7.36 \%$ del territorio nacional. La provincia se encuentra a una altitud sobre el nivel del mar que oscila entre los 0 y 500 metros (Cevallos, 2012), y presenta la mayor población ganadera del país con 977 140 cabezas (Instituto Nacional de Estadísticas y Censo, 2012).

El estudio se desarrolló en parroquias de los cantones Chone, El Carmen, Sucre, Jama, Bolívar, Tosagua y Junín, de la zona centro-norte de la provincia (Figura 1).

\section{Diseño del Estudio}

Para el análisis de riesgo individual se realizó un estudio epidemiológico transversal, donde se muestrearon 2369 bovinos en 168 hatos, con una población mayor de 5 animales. Se muestreó como promedio el $30 \%$ de los animales sanos mayores de 6 meses y se aplicó una encuesta epidemiológica para analizar la asociación entre la afectación de los animales por brucelosis y los factores de riesgo.

El tamaño de la muestra $(n=869)$ se determinó según lo establecido por Lwanga y Lemeshow (1991) para este tipo de estudio, utilizando la fórmula $n=\mathrm{Z}^{2 *} p *(l-p) / d^{2}$, donde $Z=1.96$ (nivel de confianza 95\%), $\mathrm{p}=0.10$ (proporción de la población prevista como afectada), $1-\mathrm{p}=0.9$ y d $=0.02$ (precisión absoluta a ambos lados de la proporción).

El análisis de riesgo asociado a la presentación de brucelosis en hatos ganaderos se realizó mediante un estudio epidemiológico de tipo ecológico, para el cual se utilizaron 20 hatos afectados por la enfermedad (casos) y 20 hatos no afectados por la enfermedad (controles).

Ambos estudios se realizaron entre noviembre de 2014 y marzo de 2015.

\section{Recolección de Datos}

Para determinar el riesgo individual se utilizó una base de datos, donde se registraron las variables sexo, edad, grupo racial y estado de gestación de cada animal.

Para el análisis de riesgo en hatos se utilizaron cuestionarios con 39 preguntas, donde principalmente se consideró la procedencia del agua de consumo, tipo de manejo del hato, propósito productivo, vacunación de los animales, investigación de los bovinos al ingresar en el hato, introducción de animales externos, procedencia del toro, existencia de registros sanitarios, asistencia veterinaria periódica, existencia de agua estancada en los potreros, cantidad de animales en el hato, forma de reproducción, uso de áreas arrendadas para el pastoreo, nivel de conocimiento de la enfermedad, presencia de animales de otras especies en el hato, nivel de conocimiento de la enfermedad del personal e historial de abortos en los hatos. 


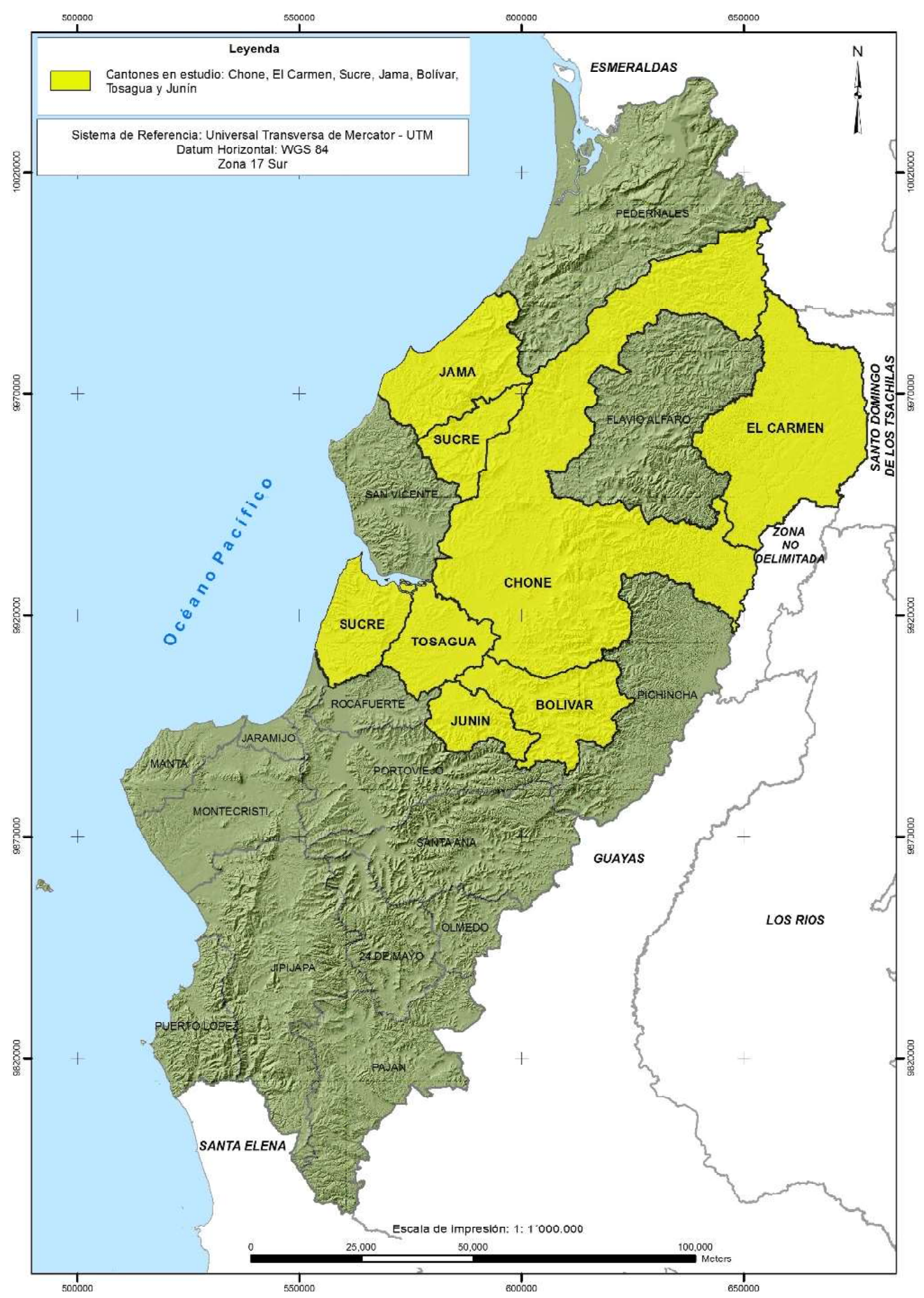

Figura 1. Mapa del área de estudio en la provincia Manabí, Ecuador 


\section{Metodología Diagnóstica}

Se tomaron $5 \mathrm{ml}$ de sangre de la vena coxígea de cada animal. Las muestras se mantuvieron entre 4 y $5 \mathrm{C}^{\circ}$ durante su traslado al laboratorio. Las muestras fueron centrifugadas y el suero resultante se almacenó en congelación entre -18 y $-20^{\circ} \mathrm{C}$.

Para el diagnóstico de la brucelosis se utilizaron técnicas recomendadas por la Organización Mundial de Sanidad Animal (OIE, 2013, Ragan et al., 2013), utilizándose Rosa de Bengala (RB) como prueba tamiz y ELISA Competitivo como prueba confirmatoria. La prueba RB se realizó en el laboratorio de la Facultad de Ciencias Veterinarias, Flor Luis Cedeño, de la Universidad Técnica de Manabí, y las muestras que resultaron positivas fueron enviadas al laboratorio acreditado para el diagnóstico de brucelosis Livexlab, Quito, para realizar la prueba confirmativa.

\section{Análisis Estadístico}

Se analizó la asociación entre la afectación de los animales por brucelosis y los factores de riesgo, para lo cual se calculó la razón de probabilidades (Odds ratio), determinándose posibles diferencias entre animales expuestos al riesgo y los no expuestos. Asimismo, se analizó la asociación entre la afectación de los hatos por brucelosis y los factores de riesgo, siguiendo la misma metodología que para el caso de los animales. Se analizó además el riesgo de abortar en los hatos positivos y negativos.

En todos los casos, se utilizó la prueba de Chi cuadrado y se consideró significativa la diferencia cuando $p<0.05$. Se utilizó el paquete estadístico en línea VassarStats.net.

\section{Aspectos Éticos}

Todos los procedimientos realizados con los animales fueron aprobados por el Comité de Ética en Investigación con Animales del Instituto de Investigación de la Universidad Técnica de Manabí. En todos los casos, los dueños de los hatos firmaron el acta de consentimiento informado.

\section{Resultados}

Del total de 2369 animales muestreados, 52 fueron positivos a la prueba Rosa de Bengala utilizada como prueba tamiz y 49 fueron confirmados como positivos mediante la prueba de ELISA competitivo, resultando en una prevalencia de $2.19 \%$.

En el estudio individual se encontró asociación estadística entre la presentación de brucelosis con la edad de los animales, siendo mayor en animales con más de 5 años ( $\mathrm{p}<0.0001$, Cuadro 1). Asimismo, hubo una asociación de la enfermedad con el sexo, presentando un mayor riesgo las hembras $(\mathrm{p}<0.05)$. No se demostró asociación de la enfermedad con el estado de gestación y el grupo racial de los animales.

En el estudio de los hatos se comprobó que los dedicados solo a la producción de leche $(\mathrm{p}<0.05)$, los que no investigan el ganado antes de ingresar a sus predios $(p<0.001)$ y los que no vacunan $(p<0.001)$ presentan un mayor riesgo de afectarse por la enfermedad (Cuadro 2). Asimismo, se observó que ningún hato donde se aplica la vacunación contra brucelosis se encontraba afectado por esta enfermedad.

Como parte del estudio se determinó también que hay mayor probabilidad de que se presenten abortos en los hatos afectados por brucelosis que en los no afectados $(\mathrm{p}<0.02$; Cuadro 3).

Otras variables evaluadas, como la procedencia del agua de consumo, tipo de manejo del hato, introducción de animales externos, procedencia del toro, existencia de registros sanitarios, asistencia veterinaria periódica, existencia de agua estancada en los potreros, cantidad de animales en el hato, forma de reproducción y uso de áreas arrenda- 
Cuadro 1. Análisis de factores de riesgo individual a brucelosis bovina en de la provincia Manabí, Ecuador (2014-2015)

\begin{tabular}{|c|c|c|c|c|c|c|}
\hline Factor de riesgo & $\begin{array}{l}\text { Total de } \\
\text { bovinos }\end{array}$ & $\begin{array}{c}\text { Afectados } \\
(\%)\end{array}$ & $X^{2}$ & Signif. ${ }^{1}$ & $\begin{array}{l}\text { Odds } \\
\text { Ratio }\end{array}$ & $\begin{array}{c}95 \% \\
\text { IC }\end{array}$ \\
\hline \multicolumn{7}{|l|}{ Sexo } \\
\hline Hembra & 2,189 & 2.2 & \multirow{2}{*}{4.11} & \multirow{2}{*}{$\mathrm{p}<0.05$} & & \multirow{2}{*}{$0.0-0.03$} \\
\hline Macho & 180 & 0.0 & & & & \\
\hline \multicolumn{7}{|l|}{ Grupo racial } \\
\hline Cebú & 17 & 0.0 & \multirow{3}{*}{0.62} & \multirow{3}{*}{ NS } & & \\
\hline Europeo & 30 & 3.3 & & & & \\
\hline Mestizo & 2,285 & 2.1 & & & & \\
\hline \multicolumn{7}{|l|}{ Gestación } \\
\hline No ge stante & 485 & 2.3 & \multirow{2}{*}{0.01} & \multirow{2}{*}{ NS } & & \\
\hline Gestante & 912 & 2.7 & & & & \\
\hline \multicolumn{7}{|l|}{ Edad (años) } \\
\hline Mayor de 5 & 906 & 3.8 & \multirow{2}{*}{20.5} & \multirow{2}{*}{$\mathrm{p}<0.0001$} & \multirow{2}{*}{3.76} & \multirow{2}{*}{$2.0-7.0$} \\
\hline Hasta 5 & 1,462 & 1.0 & & & & \\
\hline
\end{tabular}

${ }^{1}$ NS $=$ No significativo

das para el pastoreo, no mostraron asociación con la presencia de brucelosis.

No se pudo evaluar el efecto del nivel de conocimiento de la enfermedad pues los dueños y el personal en el $93 \%$ de los hatos no tenían conocimiento de la misma. Tampoco se pudo evaluar la presencia de animales de otras especies en el hato como factor de riesgo pues no hubo presencia de otra especie animal.

\section{Discusión}

Se comprobó que tres de los 52 animales positivos a brucelosis por la prueba Rosa de Bengala fueron negativos con la prueba ELISA, aspecto que coincide con lo descrito por el OIE (OIE, 2013), donde se señala que una buena estrategia diagnóstica de la enfermedad debe incluir el uso de pruebas apro- badas internacionalmente para este fin y recomienda que las reacciones positivas deben volverse a comprobar con una estrategia confirmativa adecuada, utilizando pruebas más específicas que permitan descartar los falsos positivos en el diagnóstico inicial y con ello reducir las pérdidas económicas del programa de control.

Internacionalmente se reconoce que existen múltiples factores que representan un riesgo para la presencia de brucelosis bovina en los rebaños. En el presente estudio se comprobó que la edad ( $>5$ años) fue un factor de riesgo. Si bien la mayor parte de los autores coinciden con estos resultados (Swai y Schoonman, 2010; Megersa et al., 2011; Borba et al., 2012; Sanogo et al., 2012; Boukary et al., 2013; Chand y Chhabra, 2013), se encuentran estudios contradictorios que señalan que la afectación por brucelosis es mayor en animales más jóvenes (Moreno 
Cuadro 2. Análisis de factores de riesgo de brucelosis bovina en hatos de la provincia Manabí, Ecuador (2014-2015)

\begin{tabular}{|c|c|c|c|c|c|c|}
\hline Factor de riesgo & $\begin{array}{c}\text { Total } \\
\text { de } \\
\text { hatos }\end{array}$ & $\begin{array}{l}\text { Afectados } \\
(\%)\end{array}$ & $X^{2}$ & Signif. ${ }^{1}$ & $\begin{array}{l}\text { Odds } \\
\text { Ratio }\end{array}$ & $\begin{array}{c}95 \% \\
\text { IC }\end{array}$ \\
\hline $\begin{array}{l}\text { Procedencia del agua de } \\
\text { consumo }\end{array}$ & & & & & & \\
\hline $\begin{array}{l}\text { Río-Vertiente } \\
\text { Pozo-Potable }\end{array}$ & $\begin{array}{l}27 \\
13\end{array}$ & $\begin{array}{l}55.6 \\
38.5\end{array}$ & 1.03 & NS & & \\
\hline $\begin{array}{l}\text { Tipo de manejo } \\
\text { Semiintensivo } \\
\text { Extensivo }\end{array}$ & $\begin{array}{l}10 \\
30\end{array}$ & $\begin{array}{l}70.0 \\
43.3\end{array}$ & 2.13 & NS & & \\
\hline $\begin{array}{l}\text { Propósito productivo } \\
\text { Leche } \\
\text { Doble propósito }\end{array}$ & $\begin{array}{c}7 \\
33\end{array}$ & $\begin{array}{l}85.7 \\
42.4\end{array}$ & 4.33 & $\mathrm{p}<0.05$ & 8.14 & $\begin{array}{r}0.88- \\
75.5\end{array}$ \\
\hline $\begin{array}{l}\text { Vacunación } \\
\text { No } \\
\text { Sí }\end{array}$ & $\begin{array}{c}34 \\
6\end{array}$ & $\begin{array}{l}58.8 \\
0.0\end{array}$ & 7.06 & $\mathrm{p}<0.001$ & & \\
\hline $\begin{array}{l}\text { In vestigación de bovinos } \\
\text { al ingreso }\end{array}$ & & & & & & \\
\hline $\begin{array}{l}\text { No } \\
\text { Sí }\end{array}$ & $\begin{array}{l}28 \\
12\end{array}$ & $\begin{array}{l}67.9 \\
8.3\end{array}$ & 11.90 & $\mathrm{p}<0.001$ & 23.22 & $\begin{array}{c}2.6- \\
208.6\end{array}$ \\
\hline $\begin{array}{l}\text { Introducción de a nimales } \\
\text { Sí } \\
\text { No }\end{array}$ & $\begin{array}{l}28 \\
12\end{array}$ & $\begin{array}{l}57.1 \\
33.3\end{array}$ & 1.90 & NS & & \\
\hline $\begin{array}{l}\text { Registros sanitarios } \\
\text { No } \\
\text { Sí }\end{array}$ & $\begin{array}{l}26 \\
14\end{array}$ & $\begin{array}{l}46.2 \\
57.1\end{array}$ & 0.44 & NS & & \\
\hline $\begin{array}{l}\text { Procedencia del toro } \\
\text { Externa } \\
\text { Del hato }\end{array}$ & $\begin{array}{l}26 \\
14\end{array}$ & $\begin{array}{l}57.7 \\
35.7\end{array}$ & 1.76 & NS & & \\
\hline $\begin{array}{l}\text { Asistencia veterinaria } \\
\text { No } \\
\text { Sí }\end{array}$ & $\begin{array}{l}13 \\
27\end{array}$ & $\begin{array}{l}69.2 \\
40.7\end{array}$ & 2.80 & NS & & \\
\hline $\begin{array}{l}\text { Agua estancada } \\
\text { Sí } \\
\text { No }\end{array}$ & $\begin{array}{l}11 \\
29\end{array}$ & $\begin{array}{l}54.6 \\
48.3\end{array}$ & 0.13 & NS & & \\
\hline $\begin{array}{l}\text { Número de bovinos } \\
5 \text { a } 50 \\
51-100 \\
>100\end{array}$ & $\begin{array}{c}20 \\
13 \\
7\end{array}$ & $\begin{array}{l}45.0 \\
53.9 \\
57.1\end{array}$ & 0.42 & NS & & \\
\hline $\begin{array}{l}\text { Tipo de reproducción } \\
\text { Monta directa (MD) } \\
\text { MD e IA }\end{array}$ & $\begin{array}{c}36 \\
4\end{array}$ & $\begin{array}{l}52.8 \\
25.0\end{array}$ & 1.11 & NS & & \\
\hline $\begin{array}{l}\text { Áreas arrendadas } \\
\text { Sí } \\
\text { No }\end{array}$ & $\begin{array}{c}3 \\
37\end{array}$ & $\begin{array}{l}66.7 \\
48.7\end{array}$ & 0.36 & NS & & \\
\hline
\end{tabular}

${ }^{1}$ NS $=$ No significativo 
Cuadro 3. Asociación entre el estado sanitario de los hatos con respecto a brucelosis bovina y la presentación de abortos en la provincia Manabí, Ecuador (20142015)

\begin{tabular}{lcccccc}
\hline Factor de riesgo & $\begin{array}{c}\text { Total de } \\
\text { hatos }\end{array}$ & $\begin{array}{c}\text { Hatos con } \\
\text { abortos } \\
(\%)\end{array}$ & $\mathrm{X}^{2}$ & Signif. & $\begin{array}{c}\text { Odds } \\
\text { Ratio }\end{array}$ & $\begin{array}{c}95 \% \\
\text { IC }\end{array}$ \\
\hline Hatos a fectados & 20 & 52.9 & & & & \\
Hatos no afectados & 20 & 20.0 & & & & \\
\hline
\end{tabular}

et al., 2002; Matope et al., 2011). En este sentido es importante tener en cuenta que en aquellos hatos afectados donde no se investiga o donde no se eliminan los reactores positivos, los animales tienen mayores probabilidades de infectarse en la medida en que se incrementa la edad.

El sexo fue un factor de riesgo en el presente estudio, donde las hembras tenían una mayor probabilidad de padecer la enfermedad. Resultados similares se reportan en otras provincias del país, (Ayala y Tobar, 2011), lo que podría atribuirse a que el macho es más resistente que la hembra (Tesfaye et al., 2011), aunque esta hipótesis es bastante cuestionable. Por otro lado, otros autores señalan que la infección es independiente del sexo (Matope et al., 2011). Se ha reportado, además, que los toros sin infección tienen muy poca probabilidad de infectarse a través de la monta (Rodríguez et al., 2005), aspecto que pudiera explicar los resultados encontrados.

Del total de 40 hatos estudiados, solo siete se dedicaban exclusivamente a la producción de leche y, de ellos, seis $(85.7 \%)$ se encontraban afectados por brucelosis, indicando que estas unidades tienen un mayor riesgo para la presentación de la enfermedad con respecto a las unidades de doble propósito; resultados que difieren de otros reportes
(Anka et al., 2014). Sin embargo, es importante tener en consideración que en hatos lecheros se encuentran animales especializados que son más susceptibles de padecer determinadas enfermedades con respecto a los animales que se utilizan en los hatos de doble propósito, donde por lo general utilizan animales mestizos.

El ingreso de un animal infectado con brucelosis en una finca libre de esta enfermedad constituye un factor de alto riesgo para la propagación de la enfermedad (Chand y Chhabra, 2013). En el 70\% de los hatos estudiados se introducen animales comprados en ganaderías, exposiciones o en ferias de la misma o de otra localidad, los cuales pudieran estar infectados con la bacteria y contagiar el nuevo hato que lo recibe, sobre todo teniendo en cuenta que en el $70 \%$ de estas fincas no hacen una debida evaluación sanitaria antes de su introducción. Estos resultados son similares a los obtenidos por Neppas (2013).

Una de las medidas más importantes para lograr el control de la brucelosis en una explotación ganadera es a través de la ejecución de un programa que contemple la vacunación (OIE, 2013). Es por ello que en Ecuador existe un Programa Nacional de Control de Brucelosis Bovina (AGROCALIDAD, 2009), cuya estrategia de control se orienta a 
la creación de una sólida inmunidad poblacional mediante la ejecución de campañas sistemáticas de vacunación contra la brucelosis. Este estudio demuestra el incumplimiento del programa ya que solo el $15 \%$ de las unidades estudiadas vacuna metódicamente a sus animales, y es justamente en estas donde no se encontraron animales infectados. Esto evidencia la eficacia de esta medida y el riesgo que se presenta en aquellas unidades que no vacunan.

La presencia de abortos es uno de los principales signos de la infección por brucelosis (Ministerio de Agricultura y Desarrollo Rural e Instituto Colombiano Agropecuario, 2010). Los resultados demuestran que hay mayor probabilidad de que se presenten abortos en aquellas ganaderías afectadas por brucelosis que en las no afectadas. Esta conclusión es similar a otros estudios de campo realizados en el país (Neppas, 2013) y en otros países (Makita et al., 2011; Boukary, 2013; Chand y Chhabra, 2013; Anka et al., 2014; Patel et al., 2014; Lindahl et al., 2014).

\section{Conclusiones}

- Se presenta un mayor riesgo individual de presentar la enfermedad en hembras y en animales mayores de 5 años.

- Se comprobó que hay un mayor riesgo de presentación de la enfermedad en hatos dedicados a la producción de leche, en los que no investigan el estado sanitario de los animales previo a su introducción a las fincas y en los que no vacunan el ganado.

- Se demostró que los hatos afectados por brucelosis presentan mayor riesgo de que se produzcan abortos.

\section{Literatura Citada}

1. AGROCALIDAD. 2009. Programa nacional de control de brucelosis bovina.
Resolución Sanitaria N.․ 025. Quito, Ecuador. [Internet]. Disponible en: http:/ /www.agrocalidad.gob.ec/agrocalidad/ images/pdfs/sanidadanimal/ programa_nacional_brucelosis_bovina.pdf

2. Anka MS, Hassan L, Bejo SK, Zainal MA, Mohamad R, Salleh A, Adzhar $A$. 2014. A case-control study of risk factors for bovine brucellosis seropositivity in peninsular Malaysia. PLoS ONE 9: e108673. doi:10.1371/ journal.pone. 0108673

3. Ayala E, Tobar L. 2011. Incidencia de brucelosis bovina (Brucella abortus) en los hatos lecheros de la Asociación Rancheros del Norte, Parroquia El Carmelo, Cantón Tulcán, Provincia del Carchi, Tesis Ingeniero en Desarrollo Integral Agropecuario. Carchi: Universidad Politécnica Estatal de Carchi. 112 p.

4. Borba MR, Stevenson MA, Gonzalves VSP, Ferreira Neto JS, Ferreira F, Amaku M, Telles EO, et al. 2013. Prevalence and risk-mapping of bovine brucellosis in Maranhão State, Brazil. Prev Vet Med 110: 169-176. doi: 10.1016/ j.prevetmed.2012.11.013

5. Boukary AR, Saegerman, C, Abatih $E$, Fretin D, Bada RA, De Deken R, Harouna HA, et al. 2013. Seroprevalence and potential risk factors for Brucella spp infection in traditional cattle, sheep and goats reared in urban, periurban and rural areas of Niger. PLOS/ ONE 8: e83175. doi: 10.1371/ journal.pone. 0083175

6. Cevallos FOF. 2012. Caracterización morfoestructural y faneróptico del bovino criollo en la provincia de Manabí. Tesis de Magíster. Los Ríos: Universidad de Córdoba. $67 \mathrm{p}$.

7. Chand P, Chhabra R. 2013. Herd and individual animal prevalence of bovine brucellosis with associated risk factors on dairy farms in Haryana and Punjab in India. Trop Anim Health Prod 45: 13131319. doi: $10.1007 / \mathrm{s} 11250-013-0362-\mathrm{y}$

8. Díaz $\boldsymbol{A E}$. 2013. Epidemiología de la brucelosis causada por Brucella melitensis, Brucella suis y Brucella 
abortus en animales domésticos. Rev Sci Tech Off Int Epiz 32: 43-51.

9. Instituto Nacional de Estadísticas y Censo. 2012. Encuesta de superficie y producción agropecuaria contínua ESPAC 2012. Quito, Ecuador. p 1-52.

10. Lwanga SK, Lemeshow S. 1991. Determinación del tamaño de las muestras en los estudios sanitarios. Manual Práctico. Ginebra: OMS. 80 p.

11. Lindahl E, Sattorov N, Boqvist S, Sattori L, Magnusson U. 2014. Seropositivity and risk factors for Brucella in dairy cows in urban and periurban small-scale farming in Tajikistan. Trop Anim Health Prod 46: 563-569. doi: 10.1007/s11250-013-0534-9

12. Makita K, Fèvre EM, Waiswa C, Eisler MC, Thrusfield M, Welburn SC. 2011. Herd prevalence of bovine brucellosis and analysis of risk factors in cattle in urban and peri-urban areas of the Kampala economic zone, Uganda. BMC Vet Res 7: 60-67. doi: 10.1186/ 1746-6148-7-60

13. Matope G, Bhebhe E, Muma J, Oloya J, Madekurozwa R, Lund A, Skjerve E. 2011. Seroprevalence of brucellosis and its associated risk factors in cattle from smallholder dairy farms in Zimbabwe. Trop Anim Health Prod 43: 975-982. doi: 10.1007/s11250-011-9794-4

14. Megersa B, Biffa D, Niguse F, Rufael T, Asmare K, Skjerve E. 2011. Cattle brucellosis in traditional livestock husbandry practice in Southern and Eastern Ethiopia, and its zoonotic implication. Acta Vet Scand 53: 24-31. doi: 10.1186/1751-0147-53-24

15. Ministerio de Agricultura y Desarrollo Rural, Instituto Colombiano Agropecuario. 2010. Brucelosis bovina. Prevención, diagnóstico y control. $4^{\mathrm{a}}$ ed. Bogotá DC, Colombia: Produmedios. $12 \mathrm{p}$.

16. Moreno JF, Rentería TB, Searcy R, Montano MF. 2002. Seroprevalencia y factores de riesgo asociados a la brucelosis bovina en hatos lecheros de
Tijuana, Baja California. Téc Pec Méx 40: 243-249.

17. Neppas M. 2013. Prevalencia de brucelosis bovina mediante la prueba de anillo en leche (Ring test) y rosa de bengala en la asociación agropecuaria el ordeño de la chimba - cantón Cayambe de la provincia Pichincha. Tesis de Ingeniero Agropecuario. Quito: Universidad Salesiana. $122 \mathrm{p}$.

18. [OIE] Organización Mundial de Sanidad Animal. 2013. Brucelosis bovina. En: Manual de las pruebas de diagnóstico y de las vacunas para los animales terrestres. Vol 1, Cap. 2.4.3. $7^{\mathrm{a}}$ ed. París: OIE. p 1-39.

19. Patel MD, Patel PR, Prajapati MG, Kanani AN, Tyagi KK, Fulsoundar $A B$. 2014. Prevalence and risk factor's analysis of bovine brucellosis in peri-urban areas under intensive system of production in Gujarat, India. Veterinary World 7: 509-516. doi: 10.14202/ vetworld.2014.509-516

20. Poulsen KP, Hutchins FT, McNulty CM, Tremblay $M$, Zabala $C$, Barragan V, López L, Trueba G, Bethe $J W .2014$. Brucellosis in dairy cattle and goats in northern Ecuador. Am J Trop Med Hyg 90: 712-715. doi: 10.4269/ ajtmh.13-0362

21. Ragan V, Vroegindewey G, Babcock S. 2013. International standards for brucellosis prevention and management. Rev Sci Tech Off Int Epiz 32: 189-198.

22. Rodríguez VY, Ramírez SW, Antúnez SG, Pérez BF, Ramírez PY, Igarza PA. 2005. Brucelosis bovina, aspectos históricos y epidemiológicos. REDVET 6(9). [Internet]. Disponible en: http:// www.veterinaria.org/revistas/redvet/ n090905/090503.pdf

23. Ron-Román J, Ron-Garrido L, Abatih E, Celi-Erazo M, Vizcaíno-Ordóñez L, Calva-Pacheco J, González-Andrade $P$, et al. 2014. Human brucellosis in northwest Ecuador: typifying Brucella spp, seroprevalence, and associated risk factors. Vector-Borne Zoonotic Dis 14: 124-133. doi: 10.1089/vbz.2012.1191 
24. Sanogo M, Abatih E, Thys E, Fretin D, Berkvens D, Saegerman C. 2012. Risk factors associated with brucellosis seropositivity among cattle in the central savannah-forest area of Ivory Coast. Prev Vet Med 107: 51-56. doi: 10.1016/ j.prevetmed.2012.05.010

25. Swai E, Schoonman L. 2010. The use of rose bengal plate test to asses cattle exposure to Brucella infection in traditional and smallholder dairy production systems of Tanga region of Tanzania. Vet Med Internat 2010: 837950. doi: 10.4061/2010/837950

26. Tesfaye $G$, Tsegaye W, Chanie $M$, Abinet F. 2011. Seroprevalence and associated risk factors of bovine brucellosis in Addis Ababa dairy farms. Trop Anim Health Prod 43: 1001-1005. doi: 10.1007/s11250-011-9798-0 